\title{
BOUNDARY BEHAVIOR OF MEROMORPHIC FUNCTIONS ALONG GREEN'S LINES
}

\author{
MIKIO NIIMURA \\ (Received 5 March 1980; revised 15 January 1981) \\ Communicated by E. Strzelecki
}

\begin{abstract}
In this note we study the boundary behavior of meromorphic functions in bounded plane regions along Green's lines. As applications we obtain extensions of Lohwater's theorems and Seidel's theorems concerning radial cluster sets.
\end{abstract}

1980 Mathematics subject classification (Amer. Math. Soc.): 30 A 72.

A. J. Lohwater has given the following analogues (1) and (2) of Iversen's theorem and Gross-Iversen's theorem concerning boundary cluster sets (see Lohwater (1960)): Let $g$ be a meromorphic function in $\{|z|<1\}$ and let $E^{*}$ be any set of measure zero on $\{|z|=1\}$. Let $C\left(g, e^{i \theta^{*}}\right)$ and $C_{R-E^{*}}\left(g, e^{i \theta^{*}}\right)$ denote the cluster set of $g$ at $e^{i \theta^{*}}$ and the radial boundary cluster set of $g$ at $e^{i \theta^{*}}$ modulo $E^{*}$, respectively.

(1) $C\left(g, e^{i \theta^{*}}\right)-C_{R-E^{*}}\left(g, e^{i \theta^{*}}\right)$ is open.

(2) Every value of $C\left(g, e^{i \theta^{*}}\right)-C_{R-E^{*}}\left(g, e^{i \theta^{*}}\right)$ is assumed by $g$ in any neighborhood of $e^{i \theta^{*}}$ with the possible exception of a set of capacity zero.

W. Seidel has given the following results (3) and (4) (see Seidel (1934)): Let $g$ be an analytic function in $\{|z|<1\}$ with $|g|<1$. Let $0<\alpha_{1}<\theta<\alpha_{2}<2 \pi$, $r=1$, be an arc of $\{|z|=1\}$ such that $\lim _{r \rightarrow 1}\left|f\left(r e^{i \theta}\right)\right|=1$ for almost all values of $\theta$ in $\left\{\alpha_{1}<\theta<\alpha_{2}\right\}$. Let $e^{i \theta^{*}}$ be a singular point of $g$ lying in $\left\{\alpha_{1}<\theta<\alpha_{2}\right\}$.

(3) $C\left(g, e^{i \theta^{*}}\right)=\{|w| \leqslant 1\}$.

(4) The range of $g$ at $e^{i \theta^{*}}$ is a set of points everywhere dense in $\{|w|<1\}$.

In this note we study the boundary behavior of meromorphic functions in bounded plane regions along Green's lines. As applications we obtain extensions 
of (1), (2), (3) and (4). Namely (1), (2), (3) and (4) are special cases of Theorems 1 and 2 , and Corollaries 1 and 2 , respectively.

For the definitions concerning Green's lines we refer to Sario and Nakai (1970). Let $D$ be a bounded region in the complex plane and let $f$ be a nonconstant meromorphic function in $D$. We consider Green's lines issuing from a fixed point chosen arbitrarily in $D$. Let $z_{0}$ be a point on the boundary $\partial D$ of $D$ taken in the Euclidean sense. We assume that $z_{0}$ satisfies the following (5) and (6):

(5) For any $r$-neighborhood $U\left(z_{0}, r\right)$ of $z_{0}, U\left(z_{0}, r\right) \cap \partial D$ is of positive harmonic measure.

(6) There exists no nondegenerate continuum $K$ containing $z_{0}$ such that every point of $K$ is an accumulation point of $\partial D-K$.

The cluster set of $f$ at $z_{0}$ is defined as

$$
C\left(f, z_{0}\right)=\bigcap_{r>0} \overline{f\left(U\left(z_{0}, r\right) \cap D\right)},
$$

where the bar denotes closure. Let $E^{\prime}$ be any set of harmonic measure zero lying in $\partial D$. It is easy to see from Theorem 8.6 and Theorem 8.7 of Arsove and Johnson (1970) that for any $U\left(z_{0}, r\right)$ there are Green's lines terminating at points of $U\left(z_{0}, r\right) \cap \partial D$ except for a set $E^{\prime \prime}$ of harmonic measure zero lying in $\partial D$. We put $E=E^{\prime} \cup E^{\prime \prime} \cup\left\{z_{0}\right\}$. Let $L\left(z^{\prime}, t\right)(0<t<1)$ denote a parametric representation of each Green's line terminating at $z^{\prime} \in \partial D$. The boundary cluster set of $f$ at $z_{0}$ along Green's lines modulo $E$ is defined as

$$
C_{R-E}\left(f, z_{0}\right)=\bigcap_{r>0}\left[\overline{\bigcup_{z^{\prime} \in W(r)} f\left(L\left(z^{\prime}, t\right)\right)}\right],
$$

where $W(r)=U\left(z_{0}, r\right) \cap \partial D-E . C_{R-E}\left(f, z_{0}\right)$ is not empty. A subregion of $D$ is called of type $S O_{H B}$ if there is no nonconstant bounded harmonic function in the subregion which vanishes continuously at every point on its relative boundary with respect to $D$.

TheOREM 1. $C\left(f, z_{0}\right)-C_{R-E}\left(f, z_{0}\right)$ is open.

Proof. We take any point $w^{*}$ of $C\left(f, z_{0}\right)-C_{R-E}\left(f, z_{0}\right)$ and choose a $U\left(w^{*}, r^{*}\right)$ and an open set $G$ with the properties that $C_{R-E}\left(f, z_{0}\right) \subset G$ and that $U\left(w^{*}, r^{*}\right) \cap G$ is empty. It is possible to find a $U\left(z_{0}, r_{0}\right)$ with the property that for each $L\left(z^{\prime}, t\right)$ terminating at $z^{\prime} \in W\left(r_{0}\right)$ there is a $t_{0}$ such that $f\left(L\left(z^{\prime}, t\right)\right) \subset G$ for all $t>t_{0}$. Let $D^{\prime}$ be a component of $f^{-1}\left(U\left(w^{*}, r^{*}\right)\right)$ with $D^{\prime} \subset U\left(z_{0}, r_{0}\right)$. The 
subset $W$ of $W\left(r_{0}\right)$ such that for $z^{\prime} \in W, L\left(z^{\prime}, t\right) \cap D^{\prime}$ is not relatively compact in $D$ is of harmonic measure zero. Hence the set of ends of Green's lines $L$ such that $L \cap D^{\prime}$ is not relatively compact in $D$ is of harmonic measure zero. Therefore by Theorem 8.7 of Arsove and Johnson (1970), the totality of regular Green's lines $L$ such that $L \cap D^{\prime}$ is not relatively compact in $D$ is of Green's measure zero. Thus by Lemma 3 of Nagasaka (1978), $D^{\prime}$ is of type $S O_{H B}$. It follows from Theorem 21.2 of Heins (1955) that $U\left(w^{*}, r^{*}\right)-f\left(D^{\prime}\right)$ is of capacity zero.

We put $V_{n}=f^{-1}\left(U\left(w^{*}, r^{*} / n\right)\right), n=1,2,3, \ldots$ Evidently $z_{0} \in \bar{V}_{n}$. If for some $N, f^{-1}\left(V_{N}\right)$ consists of infinitely many components $\left\{D_{n}\right\}$ such that either $z_{0} \in \bar{D}_{n}$ for every $n$ or $z_{0} \notin \bar{D}_{n}$ for every $n$, then $\left\{D_{n}\right\}$ converges to $z_{0}$. Otherwise the following two possibilities would occur: One is the case that for a subregion $D_{n}^{\prime}$ of each $D_{n}$, an infinite subsequence $\left\{D_{k}^{\prime}\right\}$ of $\left\{D_{n}^{\prime}\right\}$ converges metrically to a nondegenerate continuum, containing $z_{0}$, of $\partial D$ (see Alexandroff and Hopf (1935), p. 111). For a sufficiently large $k^{*}, D_{k^{*}}^{*}$ meets at least one $L\left(z^{\prime}, t\right)\left(t>t_{0}\right)$ terminating at $z^{\prime} \in W\left(r_{0}\right)$. This is a contradiction. The other is the case that infinitely many level curves $\left|f(z)-w^{*}\right|=r^{*} / N$ meet a disc contained in $D$. This is also a contradiction (see Collingwood and Lohwater (1966), p. 137). It is therefore seen that $U\left(w^{*}, r^{*}\right) \subset C\left(f, z_{0}\right)$.

If $f^{-1}\left(V_{n}\right)$ consists of only finitely many components $\left\{D_{n, k}\right\}_{k}$ for each $n$, then we can choose a component $G_{n}=D_{n, k}$ with $z_{0} \in \bar{G}_{n}$ for each $n$ and obtain a sequence $\left\{G_{n}\right\}$ with $G_{n+1} \subset G_{n}$. $\left\{G_{n}\right\}$ converges to $z_{0}$. Otherwise $\cap \bar{G}_{n}$ would contain a nondegenerate continuum lying in $D$. Indeed if there is a nondegenerate continuum, containing $z_{0}$, of $\partial D$, then there are Green's lines $L\left(z^{\prime}, t\right)$ each terminating at $z^{\prime} \in W\left(r_{0}\right)$ such that for all $t>t_{0}, G_{1} \cap L\left(z^{\prime}, t\right)$ is empty. This is impossible since $f$ is nonconstant.

Consequently there is a simple curve $L^{*}$ lying in $G_{1}$ and terminating at $z_{0}$ along which $f$ has an asymptotic piont $w^{*}$. We put $D^{*}=G_{1}-L^{*}$ and note that $D \cap \partial G_{1}$ accumulates to $z_{0}$. We may assume that $D^{*}$ is a region contained in $U\left(z_{0}, r_{0}\right)$. Hence $D^{*}$ is of type $S O_{H B}$. Therefore it is easy to see from the proof of Lemma 3 of Niimura (1979) that the following result holds: If $v$ is a bounded subharmonic function in $D^{*}$, then

$$
\varlimsup_{z \rightarrow z_{0}} v(z) \leqslant \varlimsup_{z^{\prime} \rightarrow z_{0}} v\left(z^{\prime}\right), \quad z \in D^{*}, \quad z^{\prime} \in D \cap \partial D^{*} .
$$

Thus it is easy to see from the proofs of Lemma 4 and the theorem of Niimura (1979) that we have a contradiction.

The assertion of Theorem 1 is proved.

THEOREM 2. Every point of $C\left(f, z_{0}\right)-C_{R-E}\left(f, z_{0}\right)$ is assumed by $f$ infinitely often in any neighborhood of $z_{0}$ with a possible exceptional set of capacity zero. 
Proof. Suppose that the assertion of Theorem 2 is false. It is easy to see from the proof of Theorem 1 of Niimura (1977) that there is a $U\left(w^{\prime}, r^{\prime}\right)$ contained in $C\left(f, z_{0}\right)-C_{R-E}\left(f, z_{0}\right)$ and having the following properties (i), (ii) and (iii):

(i) There is a closed set $F$ relative to $C\left(f, z_{0}\right)-C_{R-E}\left(f, z_{0}\right)$ such that for every $n, F \cap U\left(w^{\prime}, r^{\prime} / n\right)$ is of positive capacity.

(ii) There are components $D_{n, k}^{*}$ of $f^{-1}\left(U\left(w^{\prime}, r^{\prime} / n\right)\right)$ for each $n$ such that no point of $F \cap U\left(w^{\prime}, r^{\prime} / n\right)$ is assumed by $f$ in $D_{n, k}^{*}$.

(iii) For each $n, z_{0} \in \overline{\cup_{k} D_{n, k}^{*} \text {. }}$

For the $U\left(z_{0}, r_{0}\right)$ in the proof of Theorem 1, it is easy to see from the proof of Theorem 1 that there is at least one component $D_{n^{\prime}, k^{\prime}} \subset U\left(z_{0}, r_{0}\right)$. Therefore as can be seen easily from the proof of Theorem $1, D_{n^{\prime}, k^{\prime}}^{*}$ is of type $S O_{H B}$ and $U\left(w^{\prime}, r^{\prime} / n^{\prime}\right)-f\left(D_{n^{\prime}, k^{\prime}}^{*}\right)$ is of capacity zero. This is a contradiction.

The assertion of Theorem 2 is proved.

We call $z_{0}$ a singular point if $C\left(f, z_{0}\right)$ contains at least one nondegenerate continuum lying in $f(D)$. The following Corollaries 1 and 2 are immediately deduced from Theorems 1 and 2, respectively:

Corollary 1. Let $z_{0}$ be a singular point and let $\partial f(D)$ be not empty. Let there be a $U\left(z_{0}, r\right)$ such that all the cluster sets of $f$ along Green's lines terminating at each point of $U\left(z_{0}, r\right) \cap \partial D$ except for a set of harmonic measure zero are contained in $\partial f(D)$. Then $C\left(f, z_{0}\right)=\overline{f(D)}$.

COROLlaRY 2. Under the hypotheses of Corollary 1, every point of $f(D)$ is assumed by $f$ infinitely often in any neighborhood of $z_{0}$ with a possible exceptional set of capacity zero.

\section{References}

P. Alexandroff and H. Hopf (1935), Topologie (Springer-Verlag, Berlin).

M. G. Arsove and G. Johnson, Jr. (1970), 'A conformal mapping technique for infinitely connected regions', Mem. Amer. Math. Soc. 91, 1-56.

E. F. Collingwood and A. J. Lohwater (1966), The theory of cluster sets (Cambridge University Press).

M. Heins (1955), 'On the Lindelö principle', Ann. of Math. 61, 440-473.

A. J. Lohwater (1960), 'On the theorems of Gross and Iversen', J. Analyse Math. 7, 209-221.

Y. Nagasaka (1975), 'Notes on Green's lines', Hokkaido Math. J. 4, 53-58.

Y. Nagasaka (1978), 'Cluster sets on Riemann surfaces', Hokkaido Math. J. 7, 169-177.

M. Niimura (1977), 'Cluster sets on open Riemann surfaces,' Proc. Amer. Math. Soc. 66, 46-48.

M. Niimura (1979), 'On boundary cluster sets', J. Reine Angew. Math. 307/308, 130-133. 
L. Sario and M. Nakai (1970), Classification theory of Riemann surfaces (Springer-Verlag, BerlinHeidelberg-New York).

W. Seidel (1934), 'On the distribution of values of bounded analytic functions', Trans. Amer. Math. Soc. 36, 201-226.

Department of Mathematics

Shibaura Institute of Technology

3-9-14, Shibaura, Minato-ku

Tokyo, Japan 